\title{
Quintessential inflation for exponential type potentials: scaling and tracker behavior
}

\author{
Llibert Aresté Saló ${ }^{1, a}$, Jaume Haro ${ }^{2, b}$ \\ ${ }^{1}$ School of Mathematical Sciences, Queen Mary University of London, Mile End Road, London E1 4NS, UK \\ ${ }^{2}$ Departament de Matemàtiques, Universitat Politècnica de Catalunya, Diagonal 647, 08028 Barcelona, Spain
}

Received: 14 October 2020 / Accepted: 24 January 2021 / Published online: 1 February 2021

(C) The Author(s) 2021

\begin{abstract}
We will show that for exponential type potentials of the form $V(\varphi) \sim e^{-\gamma \varphi^{n} / M_{p l}^{n}}$, which are used to depict quintessential inflation, the solutions whose initial conditions take place during the slow roll phase in order to describe correctly the inflationary period, do not belong for large values of the parameter $n$ to the basin of attraction of the scaling solution - a solution of the scalar field equation whose energy density scale as the one of the fluid component of the universe during radiation or the matter domination period -, meaning that a late time mechanism to exit this behavior and depict correctly the current cosmic acceleration is not needed. However, in such cases, namely $n$ large enough, these potentials cannot correctly depict the current cosmic acceleration. This is the reason why the potential must be improved introducing another parameter -as the one in the well-known PeeblesVilenkin quintessential inflation model, which depends on two parameters, one to describe inflation and the other one to correctly depict the present accelerated evolution - able to deal with the late time acceleration of our universe.
\end{abstract}

\section{Contents}

1 Introduction ................ . . 1

2 A quintessential inflation model ......... . 2

2.1 Dynamical evolution of the scalar field . . . . . 4

2.2 Numerical simulation during radiation . . . . 5

3 A viable model . . . . . . . . . . . . 5

3.1 Numerical calculations ......... 6

4 Concluding remarks ............ 7

References .............. 7

a e-mail: 1.arestesalo@qmul.ac.uk (corresponding author)

b e-mail: jaime.haro@upc.edu

\section{Introduction}

Quintessence [1-7] is a theory used to reproduce the current cosmic acceleration without the need of a cosmological constant. In quintessence it has been shown that, for exponential potentials $V(\varphi) \sim e^{-\gamma \varphi / M_{p l}}$ with $\gamma>2$, there exists a solution whose energy density scales as the one of radiation [8]. Other successful quintessential inflation models have been found as well with potentials of similar behavior, such as the one in [9]. And it has recently been proved that for more general exponential potentials $V(\varphi) \sim e^{-\gamma \varphi^{n} / M_{p l}^{n}}$ there also exists an approximate scaling solution [10,11]. Such solution, termed as scaling solution (see [12] for a detailed classification of the potentials that lead to scaling solutions), is important in order to deal with the coincidence problem because, due to the attractor behavior of the scaling solution, if the scalar field is at the beginning of radiation in the basin of attraction of this scaling solution, it evolves as a radiation fluid. Therefore, since in standard quintessence we have two fields, the inflaton (which vanishes after releasing its energy when it oscillates in the deep well of the potential) and the quintessence scalar field, one can assume initial conditions for this field which lead it to enter into the basin of attraction of the scaling solution.

However, so that the universe enters in the late time accelerated phase, the quintessence field has to leave the scaling behavior, which could be done in several ways. Taking into account that for $0<\gamma<\sqrt{2}$ during the matter domination era there exists a tracker solution $[2,8,13,14]$ leading to an accelerating late time universe, one could add to the potential the term $e^{-\gamma \varphi / M_{p l}}$, with $0<\gamma<\sqrt{2}$. In this situation, it can be shown that the first term of the potential dominates during the radiation dominated era and the second term dominates during the matter dominated one [4]. Alternatively, one could introduce a non-minimal coupling between the quintessence field and massive neutrinos, whose effect is to modify the 
potential in the matter domination era $[10,11]$, but in that case, as we will see, the current cosmic acceleration is due to an effective cosmological constant.

On the contrary, in quintessential inflation [15-25] there is only one scalar field - the inflaton - driving the evolution of the universe by depicting both the early- and late-acceleration of the universe. Due to the attractor behavior of inflation, the initial condition of the scalar field has to be taken to belong to the basin of attraction of the slow roll solution. Then, using a quintessential inflation model based on the exponential type potentials proposed in [11], where the authors showed that there exists an approximately scaling solution, we will show that for large values of the parameter $n$ at the beginning of the radiation era the scalar field is not in the basin of attraction of the scaling solution. In fact, the value of the effective Equation of State $(\mathrm{EoS})$ parameter for the inflaton field is 1 during the radiation epoch, that is, it does not scale as the relativistic plasma whose energy density dominates during this period. As a consequence, in such cases a mechanism to exit the scaling behavior is not needed. The only thing needed to reproduce the evolution of the universe, as Peebles and Vilenkin shown in their seminal paper [15], is an inflationary potential leading to a spectral index $\left(n_{s}\right)$ and a ratio of tensor-to-scalar perturbations $(r)$ entering into the two dimensional marginalized joint confidence contour at $2 \sigma$ confidence-level (CL) provided by Planck data [26,27] combined with a quintessence potential which is dominant at late times in order to correctly depict the current cosmic acceleration.

The paper is organized as follows. In Sect. 2, we study the exponential type potentials introduced in [10], we calculate the reheating temperature of the universe using the mechanism of instant preheating [28,29] (see [30] for more details) because the potential is very smooth and the gravitational particle production of neither light nor superheavy particles is effective for this kind of potentials [31-35]. With this reheating temperature we compute the evolution of the inflaton field during the kination regime [36] in order to obtain its initial conditions at the beginning of the radiation epoch. Finally, with this initial data we integrate numerically the dynamical system to show that for $n$ sufficiently large (we have taken the value of $n=10$ to carry out the computations) the dynamics of the inflaton field is completely different to the one of the scaling solution. Section 3 is devoted to the study of a viable model of quintessential inflation whose potential is the combination of an exponential type potential - which stands for inflation - with a pure exponential potential which will reproduce the late time acceleration of the universe. To obtain numerically the value of the parameter on which the model depends we use the current observation data such as the red-shift at the beginning of the matter-radiation equality, the current values of the Hubble rate and the ratio of the matter energy density to the critical one. Finally, in Sect. 4 we present the conclusions of our work.

The units used throughout the paper are $\hbar=c=1$ and we denote the reduced Planck's mass by $M_{p l} \equiv \frac{1}{\sqrt{8 \pi G}} \cong$ $2.44 \times 10^{18} \mathrm{GeV}$.

\section{A quintessential inflation model}

In this work we will consider the same Exponential Inflationtype potentials studied, for the first time, in [10],

$V(\varphi)=V_{0} e^{-\lambda \varphi^{n} / M_{p l}{ }^{n}}$,

where $\lambda$ is a dimensionless parameter and $n$ is an integer.

For this model the power spectrum of scalar perturbations, its spectral index and the ratio of tensor to scalar perturbations are given by (see for details of the calculations [11])

$$
\begin{aligned}
& \mathcal{P}=\frac{V_{0} e^{-\lambda \varphi^{n} / M_{p l}{ }^{n}}}{12 \pi^{2} n^{2} \lambda^{2} M_{p l}^{6-2 n} \varphi^{2 n-2}} \sim 2 \times 10^{-9}, \\
& n_{s}=1-n \lambda\left(\frac{\varphi}{M_{p l}}\right)^{n-2}\left(2 n-2+\lambda n\left(\frac{\varphi}{M_{p l}}\right)^{n}\right)
\end{aligned}
$$

and

$r=8 n^{2} \lambda^{2}\left(\frac{\varphi}{M_{p l}}\right)^{2 n-2}$.

An important relation is obtained combining the Eqs. (3) and (4),

$\lambda\left(\frac{\varphi}{M_{p l}}\right)^{n}=\frac{r(2 n-2)}{n\left(8\left(1-n_{s}\right)-r\right)}$,

which leads to the formula for the power spectrum

$\mathcal{P}=\frac{2 V_{0} e^{-\frac{r(2 n-2)}{n\left(8\left(1-n_{S}\right)-r\right)}}}{3 \pi^{2} M_{p l}^{4} r} \sim 2 \times 10^{-9}$

and, thus,

$V_{0} \sim 3 \pi^{2} r e^{\frac{r(2 n-2)}{n\left(8\left(1-n_{s}\right)-r\right)}} \times 10^{-9} M_{p l}^{4}$,

which for the viable values of $n_{s}=0.9649$ (the central value of the spectral index) and $r=0.02$ leads to

$V_{0} \sim 6 \pi^{2}(1.166)^{\frac{n-1}{n}} \times 10^{-11} M_{p l}^{4}$.

It is important to realize that a way to find theoretically the possible values of the parameter $\lambda$ is to combine the Eqs. (4) and (5) to get

$\lambda=\left(\frac{n\left(8\left(1-n_{s}\right)-r\right)}{2 r(n-1)}\right)^{n-1}\left(\frac{r}{8 n^{2}}\right)^{n / 2}$.

And, using the theoretical values $r \leq 0.1$ and $n_{s}=0.9649 \pm$ 0.0042 (see for instance [26,27]), one can find the candidates of $\lambda$ at $2 \sigma \mathrm{CL}$. These values have to be checked for the joint 
contour in the plane $\left(n_{s}, r\right)$ at $2 \sigma \mathrm{CL}$, when the number of efolds is approximately between 60 and 75, which is what happens in quintessential inflation due to the kination phase $[21,37]$ - the energy density of the scalar field is only kinetic $[36,38]$ - after the inflationary period.

For this kind of potentials, in order to compute the number of efolds we need to calculate the main slow-roll parameter

$\epsilon=\frac{M_{p l}^{2}}{2}\left(\frac{V_{\varphi}}{V}\right)^{2}=\frac{\lambda^{2} n^{2}}{2}\left(\frac{\varphi}{M_{p l}}\right)^{2 n-2}$,

whose value at the end of inflation is $\epsilon_{E N D}=1$, meaning that at the end of this epoch the field reaches the value $\varphi_{E N D}=$ $\left(\frac{2}{n^{2} \lambda^{2}}\right)^{\frac{1}{2 n-2}} M_{p l}$.

Then, the number of efolds is given by

$$
\begin{aligned}
N & =\frac{1}{M_{p l}} \int_{\varphi}^{\varphi_{E N D}} \frac{1}{\sqrt{2 \epsilon}} d \varphi \\
& =\frac{1}{n \lambda(n-2)}\left[\left(\frac{\varphi}{M_{p l}}\right)^{2-n}-\left(\frac{2}{n^{2} \lambda^{2}}\right)^{\frac{2-n}{2 n-2}}\right]
\end{aligned}
$$

and, thus, combining the Eqs. (3), (4) and (11) one obtains the spectral index and the tensor/scalar ratio as a function of the number of efolds and the parameter $\lambda$.

On the other hand, since inflation ends at $\varphi_{E N D}=$ $\left(\frac{2}{n^{2} \lambda^{2}}\right)^{\frac{1}{2 n-2}} M_{p l}$, when the effective Equation of State (EoS) parameter is equal to $-1 / 3$, meaning that $\dot{\varphi}_{E N D}^{2}=V\left(\varphi_{E N D}\right)$, the energy density at the end of inflation is

$$
\begin{aligned}
& \rho_{\varphi, E N D}=\frac{3}{2} V\left(\varphi_{E N D}\right) \\
& \quad=9 \pi^{2}(1.166)^{\frac{n-1}{n}} e^{-\lambda\left(\frac{2}{n^{2} \lambda^{2}}\right)^{\frac{n}{2 n-2}}} \times 10^{-11} M_{p l}^{4}
\end{aligned}
$$

and the corresponding value of the Hubble rate is given by

$H_{E N D}=\sqrt{\frac{3}{10}} \pi(1.166)^{\frac{n-1}{2 n}} e^{-\frac{\lambda}{2}\left(\frac{2}{n^{2} \lambda^{2}}\right)^{\frac{n}{2 n-2}}} \times 10^{-5} M_{p l}$,

which will constrain very much the values of the parameter $\lambda$ because in all viable inflationary models at the end of inflation the value of the Hubble rate is of the order of $10^{-6} M_{p l}$ [39]. In fact, when (13) is of the order $10^{-6} M_{p l}$ one gets

$$
\lambda \sim \frac{\left(2 / n^{2}\right)^{n / 2}}{\left[\ln \left(30 \pi^{2}(1.166)^{(n-1) / n}\right)\right]^{n-1}} .
$$

Then, to perform numerical calculations, throughout the paper we will use the values of $n=10$ and $r=0.02$ and, thus, for $\lambda=4.1 \times 10^{-16}$ we have obtained approximately 67.4 efolds, which is a viable value in quintessential inflation. Note that the only constraint for $r$ is $r \leq 0.1$ and, therefore, we have been able to use the value which enables Eqs. (9) and (14) to be compatible one to another, which turns out to be $r=0.02$ for $n=10$. With these values, if the particles are created via instant preheating [28,29] - which seems the best mechanism due to the smoothness of the potential - we have to obtain the Enhanced Symmetry Point (EPS), which is the value of the field at which its temporal derivative is maximum. In our case, taking initial conditions during the slow roll period (recall that the slow roll solution is an attractor, so the evolution of the inflation field is the same for all initial conditions in the basin of attraction of the slow roll solution) we have obtained by integrating numerically the dynamical system that approximately $\dot{\varphi}_{\max } \cong 2.9 \times 10^{-6} M_{p l}^{2}$ at $\varphi_{\max } \cong 37 M_{p l}$.

After this, we have to find out the moment when kination starts, which could be chosen at the moment when the effective EoS parameter is very close to 1 . Assuming for instance that kination starts at $w_{\text {eff }} \cong 0.99$, we have numerically obtained $\varphi_{k i n} \cong 47 M_{p l}$ and $\dot{\varphi}_{k i n} \cong 5.6 \times 10^{-9} M_{p l}^{2}$, and hence $H_{k i n} \cong 2.3 \times 10^{-9} M_{p l}$.

On the other hand, since in instant preheating the effective mass of the quantum field $\chi$ field coupled with the inflaton $\varphi$ is given by $g\left(\varphi-\varphi_{\max }\right)$, where $g$ is the dimensionless coupling constant, at the beginning of kination the energy density of the created superheavy particles is given by

$$
\begin{aligned}
& \rho_{\chi, k i n}=g\left(\varphi_{k i n}-\varphi_{\max }\right) n_{\chi, \max }\left(\frac{a_{\max }}{a_{k i n}}\right)^{3} \\
& =10 g M_{p l} n_{\chi, \max }\left(\frac{a_{\max }}{a_{k i n}}\right)^{3},
\end{aligned}
$$

where the density of produced particles is [29]

$n_{\chi, \text { max }}=\frac{g^{3 / 2} \dot{\varphi}_{\text {max }}^{3 / 2}}{8 \pi^{3}} \cong 1.99 \times 10^{-11} g^{3 / 2} M_{p l}^{3}$

and

$$
\begin{aligned}
\left(\frac{a_{\max }}{a_{k i n}}\right)^{3} & =e^{-3 \int_{t_{\max }}^{t_{\text {kin }}} H(t) d t} \\
& =e^{-3 \int_{\varphi_{\max }}^{\varphi_{\text {min }}} \frac{H}{\dot{\varphi}} d \varphi} \cong 1.2 \times 10^{-12}
\end{aligned}
$$

has been calculated numerically.

Then, at the beginning of the kination regime we have

$$
\begin{aligned}
& \rho_{\chi, k i n}=2.39 \times 10^{-22} g^{5 / 2} M_{p l}^{4} \text { and } \\
& \rho_{\varphi, k i n}=1.59 \times 10^{-17} M_{p l}^{4}
\end{aligned}
$$

and, denoting by $\Gamma$ the decay rate - the superheavy particles must decay into lighter ones in order to obtain a relativistic plasma needed to match with the hot Friedmann universe -, using that $\frac{H_{d e c}}{H_{k i n}}=\frac{\Gamma}{H_{k i n}}=\left(\frac{a_{k i n}}{a_{d e c}}\right)^{3}$ and taking into account that the inflaton field $\varphi$ is nearly frozen during kination, we get

$\rho_{\varphi, d e c}=3 \Gamma^{2} M_{p l}^{2}$ and $\rho_{\chi, d e c} \cong 1.04 \times 10^{-13} g^{5 / 2} M_{p l}^{3} \Gamma$. 
In addition, in order to avoid a second inflationary phase we have to impose that the decay takes place before the end of the kination [29] (see also [40] for a detailed explanation), i.e., we have to assume $\rho_{\chi, \text { dec }} \leq \rho_{\varphi, \text { dec }}$, which leads to the following constraint,

$\Gamma \geq 3.47 \times 10^{-14} g^{5 / 2} M_{p l}$.

Then, following for example Section II of [40], the reheating temperature is given by

$$
\begin{aligned}
& T_{r h}=\left(\frac{30}{\pi^{2} g_{r h}}\right)^{1 / 4} \rho_{\chi, d e c}^{1 / 4} \sqrt{\frac{\rho_{\chi}, d e c}{\rho_{\varphi, d e c}}} \\
& \cong 4.34 \times 10^{-11} g^{15 / 8}\left(\frac{M_{p l}}{\Gamma}\right)^{1 / 4} M_{p l},
\end{aligned}
$$

where $g_{r h}=106.75$ are the degrees of freedom for the Standard Model.

And, from the bound (20), we get the maximum value of the reheating temperature as

$T_{r h} \leq 1.01 \times 10^{-7} g^{5 / 4} M_{p l}=2.45 \times 10^{11} g^{5 / 4} \mathrm{GeV}$.

On the other hand, as we have already explained, the decay must be before the end of the kination phase, meaning that $\Gamma \leq H_{k i n} \cong 2.3 \times 10^{-9} M_{p l}$, which leads to the lower bound $T_{r h} \geq 6.27 \times 10^{-9} g^{15 / 8} M_{p l}=1.53 \times 10^{10} g^{15 / 8} \mathrm{GeV}$.

Moreover, to preserve the $\mathrm{BBN}$ success the reheating temperature has to be approximately constrained between $1 \mathrm{MeV}$ and $10^{9} \mathrm{GeV}$ [41], so we get the bound

$3.08 \times 10^{-12} \leq g \leq 0.23$.

Finally, to fix the reheating temperature we choose the following compatible values of the parameters, $g=10^{-2}$ and $\Gamma=10^{-12} M_{p l}$, obtaining a reheating temperature of

$T_{r h} \cong 7.72 \times 10^{-12} M_{p l} \cong 1.88 \times 10^{7} \mathrm{GeV}$.

\subsection{Dynamical evolution of the scalar field}

Next, we want to calculate the value of the scalar field and its derivative at the reheating time. Analytical calculations can be done disregarding the potential during kination because during this epoch the potential energy of the field is negligible. Then, since during kination one has $a \propto t^{1 / 3} \Longrightarrow H=$ $\frac{1}{3 t}$, using the Friedmann equation the dynamics in this regime will be

$$
\begin{aligned}
\frac{\dot{\varphi}^{2}}{2}=\frac{M_{p l}^{2}}{3 t^{2}} & \Longrightarrow \dot{\varphi}=\sqrt{\frac{2}{3}} \frac{M_{p l}}{t} \\
\Longrightarrow \varphi(t) & =\varphi_{k i n}+\sqrt{\frac{2}{3}} M_{p l} \ln \left(\frac{t}{t_{k i n}}\right) .
\end{aligned}
$$

Thus, at the reheating time, i.e., at the beginning of the radiation phase, one has

$\varphi_{r h}=\varphi_{k i n}+\sqrt{\frac{2}{3}} M_{p l} \ln \left(\frac{H_{k i n}}{H_{r h}}\right)$.

And, using that at the reheating time (i.e., when the energy density of the scalar field and the one of the relativistic plasma coincide) the Hubble rate is given by $H_{r h}^{2}=\frac{2 \rho_{r h}}{3 M_{p l}^{2}}$, one gets

$$
\begin{aligned}
& \varphi_{r h}=\varphi_{k i n}+\sqrt{\frac{2}{3}} M_{p l} \ln \left(\frac{H_{k i n}}{\sqrt{\frac{\pi^{2} g_{r h}}{45} \frac{T_{r h}^{2}}{M_{p l}}}}\right) \text { and } \\
& \dot{\varphi}_{r h}=\sqrt{\frac{\pi^{2} g_{r h}}{15}} T_{r h}^{2},
\end{aligned}
$$

where we have used that the energy density and the temperature are related via the formula $\rho_{r h}=\frac{\pi^{2}}{30} g_{r h} T_{r h}^{4}$, where the number of degrees of freedom for the Standard Model is $g_{r h}=106.75$ [42].

As we have already commented, we will take as the reheating temperature $T_{r h} \cong 2 \times 10^{-7} \mathrm{GeV}$. Then, at the beginning of the radiation era we will have

$\varphi_{r h} \cong 71.3 M_{p l} \quad \dot{\varphi}_{r h} \cong 4.99 \times 10^{-22} M_{p l}^{2}$.

To calculate the value of the field and its derivative at the matter-radiation equality, namely $\varphi_{e q}$ and $\dot{\varphi}_{e q}$, we continue assuming that the potential is negligible (this situation has to be verified numerically integrating the full dynamical system, which we will show in the next subsection), i.e., we are assuming that the effective EoS parameter of the field $w_{\varphi}=$ $\left(\dot{\varphi}^{2} / 2-V\right) /\left(\dot{\varphi}^{2} / 2+V\right)=1$, namely that the field never reaches the basin of attraction of the scaling solution (which will be proved numerically in the next subsection), which should have during the radiation epoch an effective EoS parameter equal to $1 / 3$ because it scales as a radiation fluid.

Now, we consider the central values obtained in [43] (see the second column in Table 4 of [43]) of the red-shift at the matter-radiation equality $z_{e q}=3365$, the present value of the ratio of the matter energy density to the critical one $\Omega_{m, 0}=$ 0.308 , and $H_{0}=67.81 \mathrm{Km} / \mathrm{sec} / \mathrm{Mpc}=5.94 \times 10^{-61} M_{p l}$. Then, the present value of the matter energy density is $\rho_{m, 0}=$ $3 H_{0}^{2} M_{p l}^{2} \Omega_{m, 0}=3.26 \times 10^{-121} M_{p l}^{4}$ and at the matterradiation equality we will have $\rho_{e q}=2 \rho_{m, 0}\left(1+z_{e q}\right)^{3}=$ $2.48 \times 10^{-110} M_{p l}^{4}=8.8 \times 10^{-1} \mathrm{eV}^{4}$. Now, using the relation at the matter-radiation equality $\rho_{e q}=\frac{\pi^{2}}{15} g_{e q} T_{e q}^{4}$ with $g_{e q}=3.36$ (see [42]), we get $T_{e q}=3.25 \times 10^{-28} M_{p l}=$ $7.81 \times 10^{-10} \mathrm{GeV}$. Thus, solving the dynamical equation $\ddot{\varphi}+\frac{3}{2 t} \dot{\varphi}=0$, one obtains

$\varphi_{e q}=\varphi_{r h}+2 \sqrt{\frac{2}{3}} M_{p l}\left(1-\sqrt{\frac{2 H_{e q}}{3 H_{r h}}}\right)$ 


$$
\begin{gathered}
=\varphi_{r h}+2 \sqrt{\frac{2}{3}} M_{p l}\left(1-\sqrt{\frac{2}{3}}\left(\frac{g_{e q}}{g_{r h}}\right)^{1 / 4} \frac{T_{e q}}{T_{r h}}\right) \\
\cong \varphi_{r h}+2 \sqrt{\frac{2}{3}} M_{p l} \cong 72.9 M_{p l} . \\
\dot{\varphi}_{e q}=\dot{\varphi}_{r h} \frac{t_{r h}}{t_{e q}} \sqrt{\frac{t_{r h}}{t_{e q}}}=\frac{4}{3} M_{p l} H_{e q} \sqrt{\frac{H_{e q}}{H_{r h}}} \\
=\frac{4 \pi}{9} \sqrt{\frac{g_{e q}}{5}}\left(\frac{g_{e q}}{g_{r h}}\right)^{1 / 4} \frac{T_{e q}^{3}}{T_{r h}} \cong 1.28 \times 10^{-17} \mathrm{eV}^{2},
\end{gathered}
$$

where once again we have used that $T_{r h} \cong 2 \times 10^{7} \mathrm{GeV}$.

\subsection{Numerical simulation during radiation}

To show numerically that during radiation the scalar field is not in the basin of attraction of the scaling solution, first of all we calculate the value of the red-shift at the beginning of the radiation epoch

$$
\begin{aligned}
& 1+z_{r h}=\frac{a_{0}}{a_{r h}}=\frac{a_{0}}{a_{e q}} \frac{a_{e q}}{a_{r h}}=\left(1+z_{e q}\right)\left(\frac{\rho_{r, r h}}{\rho_{r, e q}}\right)^{1 / 4} \\
& =\left(1+z_{e q}\right)\left(\frac{g_{r h}}{g_{e q}}\right)^{1 / 4} \frac{T_{r h}}{T_{e q}},
\end{aligned}
$$

where we have used that $\rho_{r, e q}=\rho_{r, r h}\left(\frac{a_{r h}}{a_{e q}}\right)^{4}$. Then, for the reheating temperature $T_{r h}=2 \times 10^{7} \mathrm{GeV}$, we get $z_{r h}=$ $-1+1.90 \times 10^{20}$.

Moreover, at the beginning of radiation the energy density of the matter will be

$$
\begin{aligned}
& \rho_{m, r h}=\rho_{m, e q}\left(\frac{a_{e q}}{a_{r h}}\right)^{3}=\rho_{m, e q}\left(\frac{\rho_{r, r h}}{\rho_{r, e q}}\right)^{3 / 4} \\
& =\frac{\pi^{2}}{30} g_{e q}\left(\frac{g_{r h}}{g_{e q}}\right)^{3 / 4} T_{r h}^{3} T_{e q} \\
& \cong 7.7 \times 10^{13} \mathrm{GeV}^{4},
\end{aligned}
$$

where we have used that $\rho_{m, e q}=\rho_{r, e q}=\rho_{e q} / 2$.

In this way, the dynamical equations after the beginning of the radiation can be easily obtained using as a time variable $N \equiv-\ln (1+z)=\ln \left(\frac{a}{a_{0}}\right)$. Recasting the energy density of radiation and matter respectively as functions of $N$, we get

$\rho_{r}(a)=\rho_{r, r h}\left(\frac{a_{r h}}{a}\right)^{4} \Longrightarrow \rho_{r}(N)=\rho_{r, r h} e^{4\left(N_{r h}-N\right)}$

and

$\rho_{m}(a)=\rho_{m, r h}\left(\frac{a_{r h}}{a}\right)^{3} \Longrightarrow \rho_{m}(N)=\rho_{m, r h} e^{3\left(N_{r h}-N\right)}$,

where $N_{r h}$ denotes the value of the time $N$ at the beginning of radiation and, as we have already obtained, $\rho_{m, r h} \cong$ $7.7 \times 10^{13} \mathrm{GeV}^{4}$ and $\rho_{r, r h} \cong 4.4 \times 10^{30} \mathrm{GeV}^{4}$.
To obtain the dynamical system for this scalar field model, we will introduce the dimensionless variables

$x=\frac{\varphi}{M_{p l}}$ and $y=\frac{\dot{\varphi}}{K M_{p l}}$,

where $K$ is a parameter that we will choose accurately in order to ease the numerical calculations. So, taking into account the conservation equation $\ddot{\varphi}+3 H \dot{\varphi}+V_{\varphi}=0$, one arrives at the following dynamical system,

$\left\{\begin{array}{l}x^{\prime}=\frac{y}{\bar{H}}, \\ y^{\prime}=-3 y-\frac{\bar{V}_{x}}{\bar{H}},\end{array}\right.$

where the prime is the derivative with respect to $N, \bar{H}=\frac{H}{K}$ and $\bar{V}=\frac{V}{K^{2} M_{p l}^{2}}$. It is not difficult to see that one can write

$\bar{H}=\frac{1}{\sqrt{3}} \sqrt{\frac{y^{2}}{2}+\bar{V}(x)+\bar{\rho}_{r}(N)+\bar{\rho}_{m}(N)}$,

where we have defined the dimensionless energy densities as $\bar{\rho}_{r}=\frac{\rho_{r}}{K^{2} M_{p l}^{2}}$ and $\bar{\rho}_{m}=\frac{\rho_{m}}{K^{2} M_{p l}^{2}}$.

Next, to integrate from the beginning of radiation up to the matter-radiation equality, i.e., from $N_{r h} \cong-50.57$ to $N_{e q} \cong$ -8.121 , we will choose $K M_{p l}=10^{-17} \mathrm{GeV}^{2}$, yielding

$$
\begin{gathered}
\bar{\rho}_{r}(N)=4.4 \times 10^{64} e^{4\left(N_{r h}-N\right)}, \\
\bar{\rho}_{m}(N)=7.7 \times 10^{47} e^{3\left(N_{r h}-N\right)}
\end{gathered}
$$

and

$\bar{V}(x)=\frac{V_{0} \times 10^{34}}{\mathrm{GeV}^{4}} e^{-\lambda x^{n}}$.

Finally, we use the initial conditions for the field as $x_{r h}=71.3$ and $y_{r h}=2.97 \times 10^{32}$ (they have been obtained in the Eq. (29). Note that we could also have used the initial conditions in $N_{r h}$ computed in (30) and (31), but the assumptions applied in this calculus might not be valid at all when including a new exponential term as done in next section. By integrating numerically the dynamical system, we conclude that, for values of the parameter $n$ greater enough, the value of the EoS parameter $w_{\varphi}$ remains 1 during radiation, namely between $N_{r h}$ and $N_{e q}$, thus proving that the inflaton field does not belong to the basin of attraction of the scaling solution in these cases.

\section{A viable model}

To depict the late time acceleration, we have to modify the original potential because it cannot explain the current observational data (for the potential (1), at the present time the density parameter $\Omega_{\varphi}=\frac{\rho_{\varphi}}{3 H^{2} M_{p l}^{2}}$ is far from its observational value, namely 0.7 ). For this reason and following the spirit of the Peebles-Vilenkin model [15], to match with the current 
observational data we have to introduce a new parameter $M$ with units of mass which must be calculated numerically. In our case, we will consider the following modification of the potential (1) by introducing a new exponential term containing the parameter $M$,

$V(\varphi)=V_{0} e^{-\lambda \varphi^{n} / M_{p l}{ }^{n}}+M^{4} e^{-\gamma \varphi / M_{p l}}$,

with $0<\gamma<\sqrt{2}$ in order to obtain that at late times the solution is in the basin of attraction of the tracker solution [13, 14], which evolves as a fluid with effective EoS parameter $w_{\text {eff }}=\frac{\gamma^{2}}{3}-1$ (see [44] for a detailed deduction of the tracker solution).

Remark 1 An important remark is in order. Our potential slightly differs from the one used in $[10,11]$

$V(\varphi)=V_{0} e^{-\lambda \varphi^{n} / M_{p l}{ }^{n}}+\left(\bar{\rho}_{\nu}-3 \bar{p}_{\nu}\right) e^{\beta \varphi / M_{p l}}$,

where $\rho_{v}=\bar{\rho}_{\nu} e^{\beta \varphi / M_{p l}}$ is the neutrino energy density with constant bare mass $m_{v}$ and $\beta>0$ is the non-minimal coupling of neutrinos with the inflaton field (see for details [11]), which has a minimum where inflation ends its evolution, thus acting as an effective cosmological constant which stands for the current cosmic acceleration. In contrast, our potential does not have a minimum and the scalar field continues rolling down the potential as happens in quintessential inflation.

On the other hand, in our case we can also justify our choice considering that the inflaton field is non-minimally coupled with neutrinos but with a negative coupling constant, namely $\beta=-\gamma<0$. Therefore, $M^{4}=\bar{\rho}_{v}-3 \bar{p}_{v}$ and the effective mass of neutrinos is given by $m_{v, \text { eff }}(\varphi)=$ $m_{v} e^{-\gamma \varphi / M_{p l}}$, which tends to zero for large values of the field. Hence, neutrinos become relativistic, contrary to what happens when $\beta$ is positive, where the neutrinos acquire a heavy mass becoming non-relativistic particles. Finally, to match with the current observational data, $M^{4}=\bar{\rho}_{v}-3 \bar{p}_{v}$ must be very small compared to the Planck's energy density. In fact, for $n=10$ and $\gamma=1$ we have numerically obtained $M^{4} \sim 10^{-4} \mathrm{MeV}^{4}$.

Now we have to solve the dynamical system (37) with initial conditions at the beginning of the radiation era. Choosing $K=H_{0}$ (the present value of the Hubble rate), the initial conditions become $x_{r h}=71.3$ and $y_{r h}=8.57 \times 10^{38}$. With this choice of the constant $K$, note that in order obtain of the parameter $M$ one has to simply impose the value of $\bar{H}=H(N) / H_{0}$ to be 1 at $N=0$.

\subsection{Numerical calculations}

Choosing for instance $\gamma=1$, integrating the dynamical system and imposing that $\bar{H}(0)=1$, we have obtained, in the case $n=10, M=1.94 \times 10^{-1} \mathrm{MeV}$. Thus, looking at formula (8), we realize that during the slow roll phase $\left(\varphi \sim M_{p l}\right)$

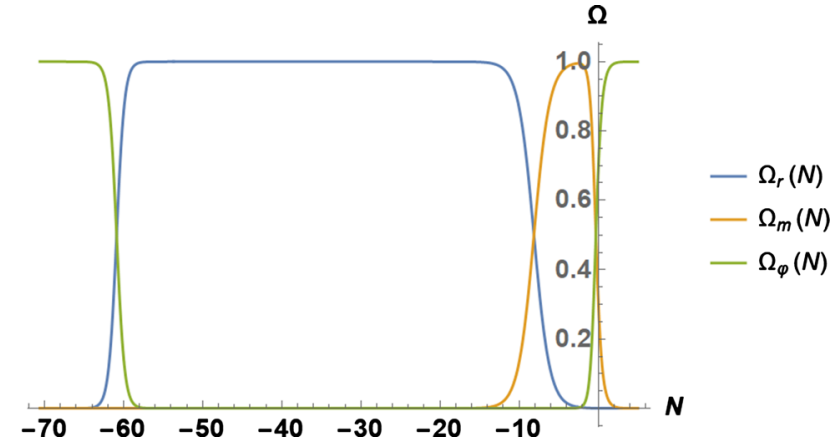

Fig. 1 The density parameters $\Omega_{m}=\frac{\rho_{m}}{3 H^{2} M_{p l}^{2}}$ (orange curve), $\Omega_{r}=$ $\frac{\rho_{r}}{3 H^{2} M_{p l}^{2}}$ (blue curve) and $\Omega_{\varphi}=\frac{\rho_{\varphi}}{3 H^{2} M_{p l}^{2}}$ (green curve), from kination to future times. To perform numerical calculations we have taken $n=10$ and $\gamma=1$

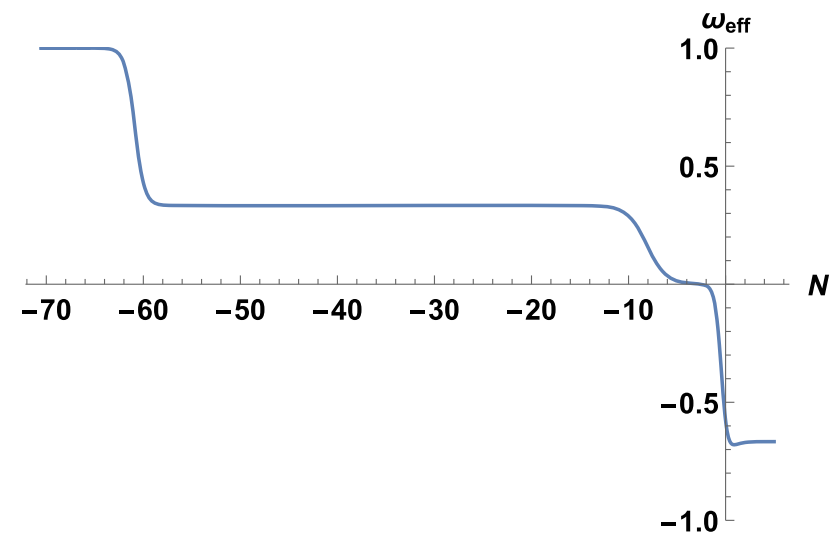

Fig. 2 The effective Equation of State parameter $w_{\text {eff }}$, from kination to future times, for $n=10$ and $\gamma=1$. As one can see in the picture, after kination the universe enters in a large period of time where radiation dominate. Then, after the matter-radiation equality, the universe becomes matter-dominated and, finally, near the present, it enters in a new accelerated phase where $w_{\text {eff }}$ approaches $\frac{1}{3}-1=-\frac{2}{3}$, that is, it has the same effective EoS parameter as the tracker solution, meaning that the solution is in the basin of attraction of the tracker one

the second term of the potential (41) is sub-leading, that is, the responsible for inflation is the first term. On the contrary, for large values of the inflaton field $\varphi\left(\varphi \sim 70 \mathrm{M}_{\mathrm{pl}}\right)$, the second term of the potential is dominant, meaning that it is the responsible for quintessence.

On the other hand, in Fig. 1 we show the evolution of the $\Omega$ 's for radiation, matter and the scalar field. One can see that the energy density of the scalar field is dominant at the present time and future. Moreover, in Fig. 2 one can deduce that the universe is accelerating because the effective EoS parameter is at the present time and future less than $-1 / 3$. 


\section{Concluding remarks}

We have shown that in quintessential inflation with exponential type potentials $V \sim e^{-\lambda \varphi^{n} / M_{p l}^{n}}$, for large values of the parameter $n$ the solutions obtained from initial conditions during the slow roll regime do not enter in the basin of attraction of the scaling solution.

In addition, we have seen that these potentials only depict the inflationary period. So, to obtain the current cosmic acceleration and describing all the evolution of the universe, we need to combine them with a quintessential potential. In our work, we have chosen as a quintessential potential an exponential potential of the type $e^{-\gamma \varphi / M_{p l}}$ with $0<\gamma<\sqrt{2}$ in order that at late times the solution is in the basin of attraction of the tracker solution, thus depicting a late time accelerated universe.

Acknowledgements This investigation has been supported by MINECO (Spain) Grant MTM2017-84214-C2-1-P, and in part by the Catalan Government 2017-SGR-247.

Data Availability Statement This manuscript has no associated data or the data will not be deposited. [Authors' comment: All data generated or analysed during this study are included in this published article [and its supplementary information files].]

Open Access This article is licensed under a Creative Commons Attribution 4.0 International License, which permits use, sharing, adaptation, distribution and reproduction in any medium or format, as long as you give appropriate credit to the original author(s) and the source, provide a link to the Creative Commons licence, and indicate if changes were made. The images or other third party material in this article are included in the article's Creative Commons licence, unless indicated otherwise in a credit line to the material. If material is not included in the article's Creative Commons licence and your intended use is not permitted by statutory regulation or exceeds the permitted use, you will need to obtain permission directly from the copyright holder. To view a copy of this licence, visit http://creativecomm ons.org/licenses/by/4.0/.

Funded by SCOAP ${ }^{3}$.

\section{References}

1. R.R. Caldwell, R. Dave, P.J. Steinhardt, Cosmological imprint of an energy component with general equation of state. Phys. Rev. Lett. 80, 1582 (1998). arXiv:astro-ph/9708069

2. B. Ratra, P.J.E. Peebles, Cosmological consequences of a rolling homogeneous scalar field. Phys. Rev. D 37, 3406 (1988)

3. P.J.E. Peebles, B. Ratra, Cosmology with a time-variable cosmological "constant". Astrophys. J. Lett. 352, L17 (1988)

4. T. Barreiro, E.J. Copeland, N.J. Nunes, Quintessence arising from exponential potentials. Phys. Rev. D 61, 127301 (2000). arXiv:astro-ph/9910214

5. S.M. Carroll, Quintessence and the rest of the world. Phys. Rev. Lett. 81, 3067 (1998). arXiv:astro-ph/9806099

6. T. Chiba, Quintessence, the gravitational constant, and gravity. Phys. Rev. D 60, 083508 (1999). arXiv:gr-qc/9903094

7. V. Sahni, L.M. Wang, A new cosmological model of quintessence and dark matter. Phys. Rev. D 62, 103517 (2000). arXiv:astro-ph/9910097
8. E.J. Copeland, M. Sami, S. Tsujikawa, Dynamics of dark energy. Int. J. Mod. Phys. D 15, 1753 (2006). arXiv:hep-th/0603057

9. D. Benisty, E.I. Guendelman, Quintessential inflation from Lorentzian slow roll. Eur. Phys. J. C 80, 577 (2020). arXiv:2006.04129 [astro-ph.CO]

10. C.Q. Geng, MdW Hossain, R. Myrzakulov, M. Sami, E.N. Saridakis, Quintessential inflation with canonical and noncanonical scalar fields and Planck 2015 results. Phys. Rev. D 92, 023522 (2015). arXiv:1502.03597 [gr-qc]

11. C.Q. Geng, C.C. Lee, M. Sami, E.N. Saridakis, A.A. Starobinsky, Observational constraints on successful model of quintessential Inflation. JCAP 1706(06), 011 (2017). arXiv:1705.01329 [gr-qc]

12. A.R. Liddle, R.J. Scherrer, A classification of scalar field potentials with cosmological scaling solutions. Phys. Rev. D 59, 023509 (1999). arXiv:astro-ph/9809272

13. P.J. Steinhardt, L.M. Wang, I. Zlatev, Cosmological tracking solutions. Phys. Rev. D 59, 123504 (1999). arXiv:astro-ph/9812313

14. L.A. Ureña-López, T. Matos, A new cosmological tracker solution for quintessence. Phys. Rev. D 62, 081302 (2000). arXiv:astro-ph/0003364

15. P.J.E. Peebles, A. Vilenkin, Quintessential inflation. Phys. Rev. D 59, 063505 (1999). arXiv:astro-ph/9810509

16. K. Dimopoulos, J.W.F. Valle, Modeling quintessential inflation. Astropart. Phys. 18, 287 (2002). arXiv:astro-ph/0111417

17. MdW Hossain, R. Myrzakulov, M. Sami, E.N. Saridakis, A class of quintessential inflation models with parameter space consistent with BICEP2. Phys. Rev. D 89, 123513 (2014). arXiv:1404.1445 [gr-qc]

18. J. Haro, J. Amorós, S. Pan, The Peebles - Vilenkin quintessential inflation model revisited. Eur. Phys. J. C 79, 505 (2019). arXiv:1901.00167 [gr-qc]

19. J. de Haro, J. Amorós, S. Pan, Simple inflationary quintessential model. Phys. Rev. D 93, 084018 (2016). arXiv:1601.08175 [gr-qc]

20. J. de Haro, E. Elizalde, Inflation and late-time acceleration from a double-well potential with cosmological constant. Gen. Relativ. Gravit. 48(6), 77 (2016). arXiv:1602.03433 [gr-qc]

21. J. de Haro, On the viability of quintessential inflation models from observational data. Gen. Relativ. Gravit. 49, 6 (2017). arXiv: 1602.07138 [gr-qc]

22. J. de Haro, J. Amorós, S. Pan, Simple inflationary quintessential model II: power law potentials. Phys. Rev. D 94, 064060 (2016). arXiv: 1607.06726 [gr-qc]

23. J. de Haro, L. Aresté Saló, Reheating constraints in quintessential inflation. Phys. Rev. D 95(5), 123501 (2017). arXiv:1702.04212 [gr-qc]

24. L. Aresté Saló, J. de Haro, Quintessential inflation at low reheating temperatures. Eur. Phys. J. C 77(11), 798 (2017). arXiv: 1707.02810 [gr-qc]

25. J. Haro, S. Pan, Bulk viscous quintessential inflation. Int. J. Mod. Phys. D 27(05), 1850052 (2018). arXiv:1512.03033 [gr-qc]

26. Y. Akrami et al., Planck 2018 results. X. Constraints on inflation. Astron. Astrophys. 641, A10 (2020). arXiv:1807.06211 [astroph.CO]

27. Y. Akrami et al., Planck 2018 results. X. Cosmological parameters. Astron. Astrophys. 641, A6 (2020). arXiv:1807.06209 [astroph.CO]

28. G. Felder, L. Kofman, A. Linde, Instant preheating. Phys. Rev. D 59, 123523 (1999). arXiv:hep-ph/9812289

29. G. Felder, L. Kofman, A. Linde, Inflation and preheating in NO models. Phys. Rev. D 60, 103505 (1999). arXiv:hep-ph/9903350

30. S. Ahmad, A. De Felice, N. Jaman, S. Kuroyanagi, M. Sami, Baryogenesis in the paradigm of quintessential inflation. Phys. Rev. D 100, 103525 (2019). arXiv:1908.03742 [gr-qc]

31. L.H. Ford, Gravitational particle creation and inflation. Phys. Rev. D 35, 2955 (1987) 
32. J. Haro, W. Yang, S. Pan, Reheating in quintessential inflation via gravitational production of heavy massive particles: a detailed analysis. JCAP 01, 023 (2019). arXiv:1811.07371 [gr-qc]

33. S. Hashiba, J. Yokoyama, Gravitational reheating through conformally coupled superheavy scalar particles. JCAP 01, 028 (2019). arXiv:1809.05410 [gr-qc]

34. D.J.H. Chung, E.W. Kolb, A. Riotto, Superheavy dark matter. Phys. Rev. D 59, 023501 (1998). arXiv:hep-ph/9802238

35. D.J.H. Chung, P. Crotty, E.W. Kolb, A. Riotto, On the gravitational production of superheavy dark matter. Phys. Rev. D 64, 043503 (2001). arXiv:hep-ph/0104100

36. M. Joyce, Electroweak baryogenesis and the expansion rate of the Universe. Phys. Rev. D 55, 1875 (1997). arXiv:hep-ph/9606223

37. A.R. Liddle, S.M. Leach, How long before the end of inflation were observable perturbations produced? Phys. Rev. D 68, 103503 (2003). arXiv:astro-ph/0305263

38. B. Spokoiny, Deflationary Universe scenario. Phys. Lett. B 315, 40 (1993). arXiv:9306008

39. A. Linde, A new inflationary universe scenario: a possible solution of the horizon, flatness, homogeneity, isotropy and primordial monopole problems. Phys. Lett. B 108, 389 (1982)
40. J. Haro, Different reheating mechanisms in quintessence inflation. Phys. Rev. D 99, 043510 (2019). arXiv:1807.07367 [gr-qc]

41. G.F. Giudice, E.W. Kolb, A. Riotto, Largest temperature of the radiation era and its cosmological implications. Phys. Rev. D 64, 023508 (2001). arXiv:hep-ph/0005123

42. T. Rehagen, G.B. Gelmini, Low reheating temperatures in monomial and binomial inflationary potentials. JCAP 06, 039 (2015). arXiv:1504.03768 [hep-ph]

43. P.A.R. Ade et al., Planck 2015 results. XIII. Cosmological parameters. Astron. Astrophys. 594, A13 (2016). arXiv:1502.01589 [astroph.CO]

44. J. Haro, J. Amorós, S. Pan, Scaling solutions in quintessential inflation. EPJC 80, 5 (2019). arXiv:1908.01516 [gr-qc] 